Editorial

\title{
Characterizing filamentous fungi from Puerto Rico for the bioremediation of organic compounds
}

\section{Editorial}

In this journal, we previously reported isolation and characterization of fungi from Puerto Rico for possible biotechnological applications. ${ }^{1}$ In order to further characterize the potential of these isolates we evaluated their ability to depolymerize organic dyes malachite green and methylene blue in reduced nutrient minimal liquid media. Growth pattern and degradation capability differences were observed amongst the isolates. These dyes are cyclic and are degraded by the extracellular lignin degrading and modifying compounds and enzymes produced by these filamentous fungi. These fungi were capable of degrading the organic dyes in aqueous environments stressed under the requirement that they must utilize the dye as their sole carbon source. These results suggest that a low cost and low maintenance water filtration system could be developed in industrial wastewater systems utilizing this whole organism, fungal degradation mechanisms.

We developed more difficult examination criteria (by the fungi's perspective) by evaluating the fungal isolates' ability to grow in minimal media with model toxins as their sole carbon source. We grew isolates on Petri plates containing minimal media plus either $0.02 \% \mathrm{v} / \mathrm{v}$ toluene or $0.02 \% \mathrm{v} / \mathrm{v}$ chlorobenzene. Surprisingly, we observed that several of our isolates exhibited healthy colony growth suggesting they were degrading the compounds as a sole carbon and micronutrient source.

Our examinations suggest there are several applications for the countless uncharacterized and underutilized fungi in Puerto Rico and elsewhere. A simplistic water filtration system utilizing fungi grown on local substrates (e.g. straw, corn cobs) should be developed. Soil hyphal penetration studies also should be conducted on these fungi along with methods of soil inoculation to develop low cost soil bioremediation techniques. In our current era of post-hurricane
Volume 7 Issue 5 - 2019

\author{
Richard L Giles \\ Departamento de Ciencias y Tecnología, Universidad \\ Interamericana de Puerto Rico, USA
}

Correspondence: Richard L Giles, Departamento de Ciencias y Tecnología, Universidad Interamericana de Puerto Rico, Recinto de Arecibo, P.O. Box 4050, Arecibo, Puerto Rico, 00614 U.S.A.,Tel+|82846|0665, Email gilesr@clevelandcc.edu

Received: September 29, 2019 | Published: October 02, 2019

Maria who devastated the island of Puerto Rico and others, the characterization and utilization of our available biological resources is more important than ever. It is essential that we catalog native organisms capable of novel biotechnological applications if not only for scientific discovery but for leveraging environmental and future financial benefits.

\section{Acknowledgments}

None.

\section{Conflicts of interest}

Author declares that there is no conflict of interest.

\section{Reference}

1. Giles RL, Babilonia LJB, Vargas DG, et al. Bioprospecting filamentous fungi from Puerto Rico for biotechnological applications. J Microbiol Exp. 2018;6(6):250-253. 